\title{
The Impact of a Care Transitions Intervention on People With Brain Injuries and Their Caregivers
}

\author{
Kristen Faye Linton \\ Chrissy Stemegna \\ Veronica Zepeda \\ Charles Watson \\ Graal Diaz \\ Thomas Duncan \\ Lauren Van Sant
}

\begin{abstract}
As our population ages, the prevalence of brain injuries increases due to strokes and falls. Brain injuries are a leading cause of rehospitalization for patients, and most brain injury survivors experience depression soon after hospital discharge. This study assessed the difference between: 1) survivors of brain injury's baseline and 30-day depression, functional ability, and quality of life and caregivers' depression and caregiver burden among those that received the Care Transitions intervention, and 2) 30-day hospital readmissions between survivors of brain injury that received Care Transitions and a control group. The study used a quasi-experimental pre-posttest design. Participants included people with brain injuries who received the Care Transitions intervention $(n=$ $22)$ and their caregivers $(n=20)$ compared to a services-as-usual control group of brain injury survivors $(n=27)$. Care Transitions is a 90-day family-focused, home visitation, coaching hospital-to-home transition intervention. Outcomes were self-reported baseline and 30-day depression, functional independence, quality of life, and caregiver burden. Hospital record data was used to report readmissions. Study results showed that there were statistically significant differences between depression, functional ability, and caregiver burden between pre- and post-survey scores among Care Transitions participants. Care transitions' participants experienced lower brain injury-related hospital readmissions than the services as usual control group. Social work hospital discharge planning needs to continue beyond the hospital and include home visitation to ensure patient and caregiver needs are met post-hospital discharge.
\end{abstract}

Keywords: Care transitions; brain injury; hospital readmission; depression; caregiver burden

Brain injury affects people of all ages. In 2014 alone 2.87 million hospitalizations, emergency department visits, and deaths combined occurred due to brain injuries in US (Centers for Disease Control [CDC], 2018). Since 2006, rates of brain injury-related emergency department visits increased by $54 \%$ (CDC, 2018). As the population ages, the prevalence of brain injuries due to a stroke or fall increases (CDC, 2018). Brain injury is a leading cause of 30-day hospital readmissions (Taylor et al., 2017). Brain injury may be caused by an internal injury (i.e., stroke) or an external injury (i.e., trauma from a fall, motor vehicle crash, or assault, etc.). Seventy percent of brain injury survivors and $20 \%$ of

\footnotetext{
Kristen Faye Linton, MSW, Ph.D., Assistant Professor, California State University Channel Islands Health Science. Camarillo, CA. Chrissy Stamegna, B.S, Veronica Zepeda, B.S., and Charles Watson, MBA, Brain Injury Center of Ventura County, Camarillo, CA. Graal Diaz, Ph.D, Thomas Duncan, D.O., and Lauren Van Sant, D.O., Ventura County Medical Center, Ventura, CA. 
their caregivers experience mild depression within the first 90 days post-hospital discharge (Kreutzer et al., 2009; Ottenbacher et al., 2001).

Traditional discharge planning, including discharge planning in the hospital as well as one follow-up phone call by hospital personnel, has not met the ongoing needs of brain injury survivors and their caregivers who desire support with healthcare system navigation, equipment and medication information, brain injury symptoms, and community resources (Linton et al., 2015). The Centers for Medicare and Medicaid's Hospital Readmissions Reduction Program (MMHRRP) lowers payments to hospitals with high 30-day readmission rates (Centers for Medicare \& Medicaid, 2020). As such, hospitals have a financial incentive to improve hospital-to-home care transitions. Effective in 2012, MMHRRP was passed in Section 3025 of the Affordable Care Act to reduce payments to inpatient prospective payment system hospitals and to avoid excess readmissions. To avoid readmissions, the needs of people with brain injuries and their caregivers must be identified and met.

Individual patients directly diagnosed with these impairments are not the only ones affected. Family caregivers take on the brunt of responsibility for ensuring that the patients' needs are met (Sander et al., 2019). According to the Bowen (1978) Family System Theory, it defines family as a relationship and emotional system in which members are interinfluenced at individual, dyadic, systemic, and intergenerational levels. Research shows that the burden of caregiving takes an immediate toll on the caregivers' mental health (Turner et al., 2010). This caregiving process starts with the patients' transition from hospital to home; about one in five caregivers experiences depression during this transitional period (Kreutzer et al., 2009; Turner et al., 2010).

Care Transitions interventions aimed at meeting the needs of patients and family caregivers have demonstrated effectiveness in improving patient outcomes among many populations (Kansagara et al., 2016). Kansagara and colleagues conducted research on mixed patient populations including older adults, patients with cancer, and those with other health conditions. However, scant research has been conducted on the impact of Care Transitions for people with brain injuries and their family caregivers (Kansagara et al., 2016).

The model of care that includes follow-up home visits with patients after hospitalization is inspired by hospital social work (Sedgwick, 2012). Hospital social workers have historically been asked to wear many hats. One-fourth of hospital social work referrals are for coping or community referral needs spanning from cleaning services for cluttered apartments to coordinating home-based recreational activities (Reckrey et al., 2014). Previous research on survivors of brain injury and their caregivers found that they desired a consistent social worker who met them in the hospital and addressed their questions after they left the hospital on a myriad of topics to further understand their brain injury, address financial and basic needs, and get referrals to community and medical services (Linton et al., 2015). However, most hospitals have a small staff of social workers who may not have adequate time to commit to all of their clients' needs, especially posthospital discharge. In many cases, patients may be referred to the hospital social worker only if they are a very high needs patient (Gibbons \& Plath, 2009). Thus, care transitions 
interventions were developed to meet the needs of patients and their caregivers that extend beyond their hospital stay.

\section{Ethnic Gaps in Care for Brain Injury Survivors}

In addition to the challenges brain injury patients and families encounter posthospitalization, there is evidence of health disparities among certain ethnic groups. Between 2005 and 2014, Latinx (Latinas and Latinos from Latin America living in the U.S.) brain-injury survivors' emergency room visit rates increased by $60.2 \%$ compared to $42.7 \%$ among white non-Latina/o patients (Hsia et al., 2018). Thirty-day hospital readmissions, defined as any unplanned hospital admission since discharge, among brain injury survivors ranged from $17-18 \%$ for Latinx and $10-14 \%$ for non-Latinx survivors (Gardener et al., 2018; Ottenbacher et al., 2001). Latinx brain injury survivors had statistically significantly higher readmission rates than white non-Latinx controlling for age and sex (Qian et al., 2013). This finding suggests that interventions aimed at addressing ethnic health disparities are important.

\section{Caregivers}

The rate of depression among caregivers of people with brain injuries is high with about $40 \%$ of caregivers of people with brain injuries experiencing depression (Loh et al., 2017). A study of patients with brain injuries and their caregivers found that caregivers had higher reports of depression during the patients' hospital-to-home transition period (the first 90 days post-hospital discharge) compared to the general population (Turner et al., 2010). Predictors of caregiver depression include social support, educational background, and relationship to the patient (Lou et al., 2015). Recent research found that there was an interdependence among caregiver and patient mental health; moreover, caregiver depression worsened as patient depression increased (Linton, 2020).

Caregivers of patients with brain injuries commonly experience caregiver burden, defined as perceived excessive care demands (Griffiths et al., 2018). There are limited studies of caregivers of people with brain injuries. One study found that one-third of family caregivers of people with dementia experienced high levels of caregiver burden (Jennings et al., 2015). Certain ethnic groups, such as Hispanics, tend to report higher levels of caregiver burden than non-Hispanic whites due to an intense cultural sense of duty to care for loved ones (Sander et al., 2019). This suggests the importance of providing culturallysensitive interventions to address caregiver burden. A pilot study of Trabajadora de Salud, a bilingual, bicultural hospital-to-home care transitions intervention designed specifically for Latinx patients with brain injuries and caregivers found that brain injury survivors and caregivers experienced improvements in depression, somatic symptoms, and caregiver burden after receiving the intervention compared to a control group that received services as usual (Linton \& Kim, 2018).

\section{Study Purpose}

Previous research on Care Transitions included only a pilot study of the intervention with a small sample of Latina/o participants (Linton \& Kim, 2018). This current study 
assessed the impact of Care Transitions among a larger sample of white non-Latina/o, Latina/o, and Asian participants representative of the community in the study area. This study assessed the impact of the Care Transitions intervention on caregiver burden and depression among a general population of caregivers of people with brain injuries. The research questions included: 1) What is the difference between survivors of brain injuries' baseline and 30-day depression, functional ability, and quality of life (QOL) among those that received a Care Transitions intervention?, 2) What is the difference between hospital readmissions among survivors of brain injuries that received Care Transitions and a services-as-usual control group?, 3) What is the difference between caregivers' baseline and 30-day depression and caregiver burden among those that received Care Transitions?

\section{Methods}

\section{Design}

The California State University Channel Islands Institutional Review Board approved this study, which used a quasi-experimental pre-posttest design. The Care Transitions intervention was delivered in person for 90-days starting once the brain injury survivor was home post-hospital discharge. Surveys were completed at the beginning of the intervention and 30 days later after the most intensive part of the intervention. The intervention includes weekly home visits for the initial 30 days, and typically bi-weekly home visits for the remaining 60 days of the intervention.

\section{Participants and Setting}

Study participants were recruited at an Adult Level II Trauma Center (TC) in a rural area on the west coast of the United States between April 2017 and October 2018. The local population was majority Latina/o and a common occupation was farm work, which is generally a low-paid profession with minimal healthcare benefits. The TC coordinator identified eligible participants and made appropriate referrals to Care Transitions coaches who visited the brain injury survivor in the hospital (for the initial visit) to inform them about the intervention. Participant inclusion eligibility criteria were as follows: 1) the participants who had a brain injury must have had a mild, moderate, or severe brain injury, 2) a planned transition from hospital to home, 3) the ability to provide consent, 4) the ability to complete pre- and post-surveys, and 5) the ability to speak English and/or Spanish. Those who participated as a caregiver must be: 1) family members of the patient, and 2) had the ability to speak English and/or Spanish. Participants who were in transition from the hospital to another institution were excluded from the study. Participation was voluntary. All participants provided written consent and chose whether to receive the Care Transitions intervention or not. Those who chose not to receive Care Transitions were placed in the services--as-usual control group. Since the research personnel had limited resources to collect data, the only outcome measured for the services-as-usual control group was hospital readmission. 


\section{Intervention and Fidelity Procedures}

The Care Transitions Intervention (CTI) was a 90-day family-focused, home visitation, coaching intervention. The original design of the intervention included only one initial home visit post-hospital discharge with weekly phone calls after the initial visit for 90 days. Care Transitions was adapted due to a pilot study conducted on Latinas/os with brain injury demonstrating improved impact in an intervention group that received all home visits, compared to a control group that received only telephone calls (Coleman, 2020; Linton \& Kim, 2018). Since Latinx are about half of the population where the study was conducted, Care Transitions was adapted to meet their unique cultural and language needs by offering services in English and Spanish, acknowledging bicultural and acculturation needs, and spending more time on personal connections and building rapport (see more in Linton \& Zepeda, 2019). All patients and their family caregivers received one hospital visit and five home visits from a Care Transitions coach and community resources. Care Transitions coaches were not trained social workers in this intervention. This coach was certified as a Brain Injury Specialist. Coaches were also bicultural, embracing and understanding both traditional American values and traditional Hispanic values. They were also bilingual, to address the unique needs of a large Hispanic population in the study location, matching previous research that has found the effectiveness of these attributes in care transitions interventions for Latinas/so/Hispanics with brain injuries (Linton \& Kim, 2018). During the hospital visit, the coach informed patients about the study and the intervention, and obtained written consent from the participants if they chose to participate. If the patient had any needs during the hospital visit, such as referrals, then the Transitions Coach provided that information. If the patient was hospitalized or in a rehabilitation facility for an extended period of time, the Transitions Coach would call to check in with the family on a weekly basis, until the patient was discharged.

The hospital medical professionals informed Care Transitions staff when the participants were ready for discharge. The coach called the participants within 72 hours to schedule an initial home visit within the first week of hospital discharge. The initial home visit included completion of the pre-survey (described under Measures), review of needs and goals for the patient and caregiver, and medication reconciliation for the patient. The medication review included collecting all medication and current prescriptions in the home. A pharmacist reviewed the list and provided a response about whether or not there were any potential medical conflicts.

Five in-person visits occurred with the patient and caregiver during the 90-day period following the initial home visit. Common patient needs included: goal-setting, goal prioritizing, stable housing, skills on gaining independence, caregiver respite, motivation, and encouragement (as reported in Linton \& Zepeda, 2019). In-person visits addressed the following topics: 1) brain injury education, 2) referrals for community resources, 3) help applying for resources and scheduling medical appointments, 4) addressing basic needs, 5) goal-setting, 6) assisting in healthcare provider communication, and 7) medication reconciliation. Coaches would commonly provide patients with homework to practice skills to gain independence. They would build rapport with patients through hands-on creative activities, such as art, taking walks, or playing sports. It was through these creative rapport-building activities that coaches would learn what motivated their patients. 
Motivation was key in supporting patients in goal-setting. Additional phone contacts varied based on the patient's needs and desires. The initial 30 days of the intervention was the most intense with weekly home visits, while the following 60 days included primarily one home visit and phone call check-ins. After the 90-day intervention period, the patient and caregiver were transitioned to ongoing support services, such as support groups, workshops, and social activities.

A random sample of case notes (10\%) from home visits were reviewed to assess feasibility, acceptability, and fidelity of the intervention; no issues were found. Certification for Brain Injury Specialists required fulfillment of a 12-hour course covering cognition, guidelines for interacting and building rapport, brain injury and behavior, medical complications, self-medication management, and families coping with brain injury. Three coaches met weekly with a supervisor and peers to "staff" their caseloads. Data on potential confounding variables were collected, such as comorbidities, receipt of formal psychological therapy, and attendance at support groups. The authors assessed the relationships between these potential confounding variables and the outcomes. No significant relationships were found.

\section{Study Outcomes and Measures}

\section{Self-Report Surveys}

Pre-surveys were administered within one week of arriving home and post-surveys were administered 30 days after the participants arrived home. Self-reported surveys were completed online or using a hard copy (depending on participant preference) and included demographic questions as well as the nine-item depression scale, the Personal Health Questionaire-9 (PHQ-9, Kroenke et al., 2001), which was distributed to all participants in the intervention group including TBI survivors and caregivers. Depression was defined as feelings of sadness, hopelessness, and a loss of interest in previously enjoyed activities (American Psychiatric Association, 2013). The PHQ-9 has been assessed for validity and reliability among people with brain injuries (Fann et al., 2005; Perrin et al., 2013; $\alpha=.86$ .90). Face and construct validity were determined by comparing the PHQ-9 to other common depression scales (Fann et al., 2005). One item on the scale asked: "Over the past two weeks, how often have you been bothered by any of the following problems? Little or no interest in doing things" with options including: not at all, several days, more than half the days, and nearly every day. All items were added to obtain a sum of the items for a total score on the PHQ-9 (potential score range $=0-29$ ). Higher scores indicated more depression. The brain injury survivors in the intervention group were also asked: 1) one item from the Functional Independence Measure ("How would you describe your overall level of functioning- physical, mental, emotional, or social from completely independent to totally dependent?"), and 2) one item on quality of life ("How do you rate your overall quality of life from very poor to very good?" with response options: very satisfied, satisfied, neutral, unsatisfied, very unsatisfied).

The caregivers' surveys also measured caregiver burden. Caregiver burden was defined as the individual's perception of problems or change in their own well-being due to their role as a caregiver (Thornton \& Travis, 2003). Four items from the Modified 
Caregiver Strain Index, which have demonstrated validity and internal and test-re-test reliability were used in the caregiver survey (potential range of scores $=0-8$; Thornton $\&$ Travis, 2003). One example item was: "I feel completely overwhelmed." Response options included: Yes, on a regular basis; yes, sometimes, and no. Higher scores indicated more caregiver burden.

\section{Hospital Readmission Data}

The TC provided data on the all-cause 30-day brain-injury related hospital readmission data among all brain injury survivor participants. No other demographic data were provided for the control group participants.

\section{Data Analysis}

To achieve an adequate power of 0.80 to test the hypothesis using a paired samples $t$ test, a sample size of 25 was calculated based on Cohen's $d$ of 0.6 and power at .80 (analysis conducted using G*Power, Faul et al., 2009). Paired samples $t$-tests were used to test the differences between self-reported pre- and post-survey scores on depression, functional ability, QOL, and caregiver burden. Analyses were conducted using IBM SPSS 18. The significance level for hypothesis testing was $\alpha=.05$. The TC's 30-day readmission data was used to compare the frequency of readmission between the intervention and control group.

\section{Results}

The total number of potential participants (identified as having a brain injury at the participating hospital) was 256; all were examined for eligibility, and a total of 120 were confirmed eligible based on eligibility criteria. A total of 50 participants consented to participate in the study, all of whom had complete hospital readmission data. Twenty-two brain injury survivors and 20 of their corresponding caregivers received Care Transitions and completed pre- and post-survey data. Twenty-seven brain injury survivors did not complete the intervention or the post-survey. Caregiver data were not complete for two of the caregivers of patients in the study. The other 27 participants were placed in the servicesas-usual control group. Descriptions of the brain injury survivors and caregivers are provided in Table 1. Brain injury survivors had an average age of $48(S D=17.61)$. The average age of caregivers was $65(S D=15.76)$. 
Table 1. Descriptives of the Brain Injury Survivors $(n=22)$ and Their Caregivers $(n=20)$

\begin{tabular}{l|r|r}
\hline \multirow{2}{*}{ Descriptives } & \multicolumn{2}{|c}{$\mathrm{n}(\%)$} \\
\cline { 2 - 3 } Gender & Survivors & Caregivers \\
Male & $14(63.6 \%)$ & $15(75.0 \%)$ \\
Female & $8(36.4 \%)$ & $5(25.0 \%)$ \\
\hline Race/Ethnicity & & \\
Latina/o, Hispanic & $11(50.0 \%)$ & $8(40.0 \%)$ \\
Non-Hispanic White & $9(40.9 \%)$ & $11(55.0 \%)$ \\
Asian & $1(4.5 \%)$ & $1(5.0 \%)$ \\
Native Hawaiian/Pacific Islander & $1(4.5 \%)$ & \\
\hline Primary Language & & \\
English & $16(72.72 \%)$ & $14(70.0 \%)$ \\
Spanish & $6(27.27 \%)$ & $5(25.0 \%)$ \\
Tagalog & & $1(5.0 \%)$ \\
\hline Insurance & & \\
Medicaid (MediCal) & $10(45.5 \%)$ & \\
Private & $5(22.7 \%)$ & \\
No Insurance & $4(18.2 \%)$ & \\
Medicare & $3(13.6 \%)$ & \\
\hline Cause of the Brain Injury & & \\
Pedestrian/Biker hit by a vehicle & $5(22.7 \%)$ & \\
Car Collision & $4(18.2 \%)$ & \\
Fall & $4(18.2 \%)$ & \\
Stroke & $3(13.6 \%)$ & \\
Attacked & $2(9.1 \%)$ & \\
Loss of Consciousness & $1(4.5 \%)$ & \\
Unknown & $3(13.6 \%)$ & \\
\hline Employment Status & $12(59.1 \%)$ & \\
Not Employed & $5(22.7 \%)$ & \\
Employed full time & $2(9.1 \%)$ & \\
Employed part time & $3(13.6 \%)$ & \\
\hline Injury Impact on Employment & $2(9.1 \%)$ & \\
Lost job due to injury & $2(9.1 \%)$ & \\
Has not impacted job & & \\
Impacted hours can work & & \\
Impacted ability to perform duties at job & & \\
\hline & & \\
\hline
\end{tabular}

The analyses revealed statistically significant improvements in depression and functional ability between pre- and post-survey scores among Care Transitions participants (Table 2). Brain injury survivors' quality of life improved but not at a statistically significant level. Care transitions' participants experienced lower brain injury related hospital readmissions than the control group ( $0 \%$ Care Transitions group compared to $7 \%$ services-as-usual control group). There were statistically significant improvements in selfreported depression and caregiver burden among the caregivers (Table 2). 
Table 2. Differences Between Baseline and 30-day Self-Report Survey Results Among Care Transitions Participants: Survivors $(n=22)$ and Caregivers $(n=20)$

\begin{tabular}{|c|c|c|c|c|c|c|}
\hline \multirow[b]{2}{*}{ Participants } & \multirow[b]{2}{*}{ Scale } & \multicolumn{2}{|c|}{$M(S D)$} & \multirow[b]{2}{*}{$t$} & \multirow[b]{2}{*}{$p$} & \multirow[b]{2}{*}{$d f$} \\
\hline & & Baseline & 30-day & & & \\
\hline \multirow[t]{3}{*}{ Survivors } & Depression $^{\mathrm{a}}$ & $17.94(6.51)$ & $14.11(5.01)$ & 2.81 & $.01 *$ & 21 \\
\hline & Functional Ability ${ }^{\mathrm{b}}$ & $2.95(0.94)$ & $2.35(1.13)$ & 2.69 & $.01 *$ & \\
\hline & Quality of Life & $3.50(0.89)$ & $3.85(0.58)$ & -1.92 & .06 & \\
\hline \multirow[t]{2}{*}{ Caregivers } & Depression $^{\mathrm{a}}$ & $14.20(5.09)$ & $11.75(1.82)$ & 2.62 & $.017 *$ & 19 \\
\hline & Burden & $12.65(4.49)$ & $10.00(3.22)$ & 3.00 & $007 *$ & \\
\hline
\end{tabular}

$* \mathrm{p}=<.05$

${ }^{\mathrm{a}}$ Higher score $=$ More Depression, ${ }^{\mathrm{b}}$ Higher score $=$ More Dependence

\section{Discussion}

This study found that Care Transitions intervention demonstrates promise in improving depression, caregiver burden, and functional ability among brain injury survivors and their caregivers. The 30-day hospital readmission rate was 7\% lower among brain injury survivors who received Care Transitions compared to a services-as-usual control group, indicating cost and time savings for those that received the Care Transitions intervention. Costs for hospitalization due to brain injury vary from $\$ 8,000$ - $\$ 33,000 /$ visit (McGarry et al., 2002). At minimum, Care Transitions potentially saved $\$ 12,000$ per patient; if $7 \%$ of the 22 Care Transitions participants had been rehospitalized, it would mean that 1.5 persons were rehospitalized (costing a minimum of $\$ 8,000$ each). In our study's project, the Care Transitions coaches were paid $\$ 15 /$ hour and worked 20 hours per week. Two care transitions coaches were employed; their monthly salaries were $\$ 2,400$ in total. There is no direct cost to the patient, as the Care Transitions intervention is free to them; this project was grant funded. In addition to preventing $7 \%$ hospital readmissions, the brain injury survivors that received Care Transitions improved in depression and functional ability, which can potentially relieve survivors' dependence on other medical systems. Chen and colleagues (2012) found that acute care only accounted for $46-65 \%$ of the medical costs survivors of brain injuries; the mean medical costs ranged from $\$ 32,000-\$ 38,000$ in the first year post-hospitalization.

Care Transitions' interventions have implications for hospital social workers, who often hold critical roles in discharge planning. Previous research has shown that patients desired hospital social workers to spend more time with them post-discharge when questions arose (Linton et al., 2015). Social workers should be supported to conduct home visits with brain injury patients post-hospital discharge, to prevent hospital readmissions. Advocacy is needed to ensure that post-hospital social work services are covered by health insurance policies, as insurance typically covers one post-hospital phone call only (see more discussion in Linton et al., 2015).

It should be noted that half of the brain injury survivors in this study self-identified as Latina/o/Hispanic, reflecting the surrounding population. Latinx brain injury survivors are more likely to experience depression symptoms than white non-Latinx (Bombardier et al., 2016). Latinx survivors reported statistically significantly higher levels of depression over time (Linton \& Kim, 2018). One study found that one in two Latinx survivors and one in 
eight white non-Latinx experienced depression (Fei et al, 2016). Functional ability is associated with depression (Ottenbacher et al., 2001). Care Transitions may have the opportunity to close gaps in disparities experienced among Latina/o/Hispanic brain injury survivors and their caregivers (Hsia et al., 2018). Since family and personal relationships are so important to Latina/o populations, the Care Transitions' intervention embraces these characteristics by supporting the whole family and conducting face-to-face home visits to develop personal relationships with the family members over time.

Family caregivers are critical in meeting the needs of patients, but their own health needs are often overshadowed by the intense needs of the patient. Family-centered care is a field in healthcare that fully acknowledges the family caregivers' need as well as the patients' needs (Goldfarb et al., 2017). Recommendations based on family-centered care include communicating with family members, family presence during medical recommendations, providing support for the family, and addressing environmental needs of the family (Davidson et al., 2017). The family-centered care philosophy also recognizes the interdependence between the health of the patient and caregiver (Linton, 2020). Care Transitions interventions need to embrace the patient and caregiver equally as both have unique needs (Linton et al., 2015). Future research should assess the impact of the Care Transitions intervention on the health status of caregivers.

Our study included limitations, such as short-term outcomes, limited sample size, inclusion of people with mild to severe brain injury, and attrition. This is the study's first publication on results from 30-day outcomes, but 90-day outcomes are being measured currently. The sample size was small, yet appropriate to gain initial data. Another weakness of our study is the lack of a control group for depression and caregiver burden. Additionally, no random assignment was conducted, nor demographic data collected on those who chose not to receive Care Transitions. Future research will include gathering data on those who choose not to receive the intervention for comparison purposes.

Given that this is a new program and enrollment occurs while patients are still in the acute care hospital setting, patients and families were often hesitant to enroll. This study was conducted in partnership with a local nonprofit, thus the decision to include people with mild to severe brain injuries arose from the nonprofit's promise to serve people with all types of brain injuries. The general brain injury recovery information and local resources needed during the hospital-to-home transition are similar for people with both mild and more severe forms of brain injury. For example, people with brain injuries and their families need to know about rehabilitation services (i.e., physical therapy and occupational therapy), mental health services, and home health care, regardless of the severity of their injury. Specific needs differ not only by brain injury severity, but also by familial resources and cultural needs. Care Transitions is a flexible intervention in that each individual home visit can be customized to meet the unique needs of the patient while maintaining an overall consistency in topics covered during the intervention for each brain injury survivor and caregiver. Reasons for low sample size and/or attrition included: missing or inaccurate contact information, patient misconception of a brain injury, or refusing to participate in the program. It should be noted that since brain injury survivors were recovering from their injury, the mere lack of outside influences may have impacted their recovery as well. Most survivors were recovering in the comfort of their own home 
during the initial 90-days post-hospitalization. The everyday stressors, such as work and driving, that influence most people were not experienced among participants, thus it may have helped to improve their mental health leading to more positive outcomes. However, research also shows that there is a direct correlation between the health of brain injury survivors and their caregivers (Linton, 2020). Outcomes for only one caregiver per family were measured in the current study; some brain injury survivors may have had more than one family caregiver. Many participants lived with other people who may have positively or negatively impacted their outcomes. Another important potential confounding variable is socioeconomic status. Participants with greater economic and social resources may have had more help in recovering informally from friends or formally from paid caregivers (Durish et al., 2018).

\section{Conclusion}

Hospitals may consider developing care transitions interventions, partnering with local organizations to conduct these interventions to improve discharge and adjustments made during patients' transition home, or ensuring sufficient staffing of hospital social workers to conduct home visits. Care Transitions, the intervention provided in this study, aimed to meet the needs of both patients and caregivers during their transitions from hospital-tohome. The intervention has the potential to save patients and hospitals time and money due to lower hospital readmissions. Hospital social work has transitioned more recently to focusing on discharge planning, but due to social workers' time limitations, very little follow-up is being conducted to ensure that patients' needs are met after they are discharged (Linton et al., 2015). Hospital social workers should advocate for hiring more social workers to ensure that sufficient time is given to conduct home visits in lieu of Care Transitions. A separate Care Transitions intervention may not be needed if hospital social workers were able to conduct home visits and support patients through their transition home. Given the small sample size and lack of control group for all outcome variables, future research should be conducted to assess the efficacy of Care Transitions on improving brain injury survivors' and caregivers' depression, functional ability, and caregiver burden as well as preventing brain injury-related hospital readmissions. Future research should also assess the impact of gender on caregiving as well as those caregivers that may be caring for young children and older adults simultaneously. When larger sample sizes are available, future research should also assess differential effects of the intervention on Latina/o/Hispanic participants as well as other populations.

\section{References}

American Psychiatric Association. (2013). Diagnostic and statistical manual of mental disorders $\left(5^{\text {th }}\right.$ ed.). Author.

Bombardier, C. H., Hoekstra, T., Dikmen, S., \& Fann, J. R. (2016). Depression trajectories during the first year after traumatic brain injury. Journal of Neurotrauma, 33(23), 21152124. https://doi.org/10.1089/neu.2015.4349

Bowen, M. (1978). Family therapy in clinical practice. Aronson.

Centers for Disease Control. (2018). Traumatic brain injury and concussion. https://www.cdc.gov/traumaticbraininjury/index.html 
Centers for Medicare \& Medicaid. (2020). Hospital readmissions reduction program (HRRP). https://www.cms.gov/Medicare/Medicare-Fee-for-ServicePayment/AcuteInpatientPPS/Readmissions-Reduction-Program

Chen, A., Bushmeneva, K., Zagorski, B., Colantonio, A., Parsons, D., \& Wodchis, W. P. (2012). Direct cost associated with acquired brain injury in Ontario. BMC Neurology, 12(76), 1-12. https://doi.org/10.1186/1471-2377-12-76

Coleman, E. (2020). About the Care Transitions Intervention $(\mathrm{C}$. https://caretransitions.org/about-the-care-transitions-intervention/

Davidson, J. E., Aslakson, R. A., Long, A. C., Puntillo, K. A., Kross, E. K., Hart, J., \& Netzer, G. (2017). Guidelines for family-centered care in the neonatal, pediatric, and adult ICU. Critical Care Medicine, 45(1), 103-128. https://doi.org/10.1097/ccm. $\underline{0000000000002169}$

Durish, C. L., Pereverseff, R. S., \& Yeates, K. O. (2018). Depression and depressive symptoms in pediatric traumatic brain injury: A scoping review. Journal of head Trauma Rehabilitation, 33(3), E18-E30. https://doi.org/10.1097/htr.0000000000000343

Fann, J. R., Bombardier, C. H., Dikmen, S., Esselman, P., Warms, C. A., Pelzer, E., \& Temkin, N. (2005). Validity of the Patient Health Questionnaire-9 in assessing depression following traumatic brain injury. Journal of Head Trauma Rehabilitation, 20(6), 501511. https://doi.org/10.1097/00001199-200511000-00003

Faul, F., Erdfelder, E., Buchner, A., \& Lang, A.-G. (2009). Statistical power analyses using G*Power 3.1: Tests for correlation and regression analyses. Behavior Research Methods, 41, 1149-1160. https://doi.org/10.3758/brm.41.4.1149

Fei, K., Benn, E. K., Negron, R., Arniella, G., Tuhrim, S., \& Horowitz, C. R. (2016). Prevalence of depression among stroke survivors: Racial-ethnic differences. Stroke, 47(2), 512-515. https://doi.org/10.1161/strokeaha.115.010292

Gardener, H., Leifheit, E. C., Lichtman, J., Wang, K., Wang, Y., Gutierrez, C. M., CilibertiVargas, M. A., Dong, C., Robichaux, M., Romano, J. G., \& Sacco, R. L. (2018). Abstract WP306: Race-ethnic disparities in 30-day readmission after ischemic stroke among Medicare beneficiaries in the Florida Puerto Rico Collaboration to Reduce Stroke Disparities (FL-PR CReSD) study. Stroke, 49(Suppl_1), AWP306. https://doi.org/ 10.1161/str.49.suppl 1.wp306

Gibbons, J., \& Plath, D. (2009). Single contacts with hospital social workers: The clients' experiences. Social Work in Health Care, 48(8), 721-735. https://doi.org/10.1080 $\underline{00981380902928935}$

Goldfarb, M. J., Bibas, L., Bartlett, V., Jones, H., \& Khan, N. (2017). Outcomes of patientand family-centered care interventions in the ICU: A systematic review and metaanalysis. Critical Care Medicine, 45(10), 1751-1761. https://doi.org/10.1097/ ccm.0000000000002624

Griffiths, A. W., Wood, A. M., \& Tai, S. (2018). The prospective role of defeat and entrapment in caregiver burden and depression amongst formal caregivers. Personality and Individual Differences, 120, 24-31. https://doi.org/10.1016/j.paid.2017.08.026 
Hsia, R. Y., Markowitz, A. J., Lin, F., Guo, J., Madhok, D. Y., \& Manley, G. T. (2018). Tenyear trends in traumatic brain injury: a retrospective cohort study of California emergency department and hospital revisits and readmissions. BMJ Open, 8(12), 1-11. https://doi.org/10.1136/bmjopen-2018-022297

Jennings, L. A., Reuben, D. B., Evertson, L. C., Serrano, K. S., Ercoli, L., Grill, J., \& Wenger, N. S. (2015). Unmet needs of caregivers of individuals referred to a dementia care program. Journal of the American Geriatrics Society, 63(2), 282-289. https://doi.org/10.1111/jgs.13251

Kansagara, D., Chiovaro, J. C., Kagen, D., Jencks, S., Rhyne, K., O'Neil, M., \& Englander, H. (2016). So many options, where do we start? An overview of the care transitions literature. Journal of Hospital Medicine, 11(3), 221-230. https://doi.org/10.1002/ jhm.2502

Kreutzer, J. S., Rapport, L. J., Marwitz, J. H., Harrison-Felix, C., Hart, T., Glenn, M., \& Hammond, F. (2009). Caregivers' well-being after traumatic brain injury: A multicenter prospective investigation. Archives of Physical Medicine and Rehabilitation, 90(6), 939946. https://doi.org/10.1016/j.apmr.2009.01.010

Kroenke, K., Spitzer, R. L., \& Williams, J. B. (2001). The PHQ-9: Validity of a brief depression severity measure. Journal of General Internal Medicine, 16(9), 606-613. https://doi.org/10.1046/j.1525-1497.2001.016009606.x

Linton, K. F. (2020). Latina/o and non-latina/o brain injury and dementia patients' and caregivers' health: An actor/partner interdependence model. Brain Impairment, 21(3), 237-246. https://doi.org/10.1017/brimp.2020.1

Linton, K. F., Ing, M. M., Vento, M. A., \& Nakagawa, K. (2015). From discharge planner to "concierge": Recommendations for hospital social work by clients. Social Work in Public Health, 30(6), 486-495. https://doi.org/10.1080/19371918.2015.1058730

Linton, K. F., \& Kim, B. J. (2018). A pilot study of Trabajadora de salud, a lay health worker intervention for Latinas/os with traumatic brain injuries and their caregivers. Disability and Health Journal, 11(1), 161-164. https://doi.org/10.1016/j.dhjo.2017.04.009

Linton, K. F., \& Zepeda, V. (2019). Trabajadora de Salud, a care transitions intervention, for Latinas/os with brain injuries: Three case examples. Human Behavior in the Social Environment, 30(3), 243-250. https://doi.org/10.1080/10911359.2019.1673273

Loh, A. Z., Tan, J. S., Zhang, M. W., \& Ho, R. C. (2017). The global prevalence of anxiety and depressive symptoms among caregivers of stroke survivors. Journal of the American Medical Directors Association, 18(2), 111-116. https://doi.org/10.1016/i.jamda.2016. $\underline{08.014}$

Lou, Q., Liu, S., Huo, Y. R., Liu, M., Liu, S., \& Ji, Y. (2015). Comprehensive analysis of patient and caregiver predictors for caregiver burden, anxiety and depression in Alzheimer's disease. Journal of Clinical Nursing, 24(17-18), 2668-2678. https://doi.org/10.1111/jocn.12870

McGarry, L. J., Thompson, D., Millham, F. H., Cowell, L., Snyder, P. J., Lenderking, W. R., $\&$ Weinstein, M. C. (2002). Outcomes and costs of acute treatment of traumatic brain 
injury. Journal of Trauma and Acute Care Surgery, 53(6), 1152-1159.

https://doi.org/10.1097/00005373-200212000-00020

Ottenbacher, K. J., Smith, P. M., Illig, S. B., Fiedler, R. C., Gonzales, V., \& Granger, C. V. (2001). Characteristics of persons rehospitalized after stroke rehabilitation. Archives of Physical Medicine and Rehabilitation, 82(10), 1367-1374. https://doi.org/10.1053/ apmr.2001.26088

Perrin, P. B., Stevens, L. F., Sutter, M., Hubbard, R., Sosa, D. M. D., Jove, I. G. E., \& Arango-Lasprilla, J. C. (2013). Exploring the connections between traumatic brain injury caregiver mental health and family dynamics in Mexico City, Mexico. Physical Medicine and Rehabilitation, 5(10), 839-849. https://doi.org/10.1016/j.pmrj.2013.05.018

Reckrey, J. M., Gettenberg, G., Ross, H., Kopke, V., Soriano, T., \& Ornstein, K. (2014). The critical role of social workers in home-based primary care. Social Work in Health Care, 53(4), 330-343. https://doi.org/10.1080/00981389.2014.884041

Qian, F., Fonarow, G. C., Smith, E. E., Xian, Y., Pan, W., Hannan, E. L., Shaw, B.A., Glance, L. G., Peterson, E. D., Eapen, Z. J., \& Hernandez, A. F., Schwamm, L. H., \& Bhatt, D. L. (2013). Racial and ethnic differences in outcomes in older patients with acute ischemic stroke. Circulation: Cardiovascular Quality and Outcomes, 6(3), 284-292. https://doi.org/10.1161/circoutcomes.113.000211

Sander, A. M., Hanks, R. A., Ianni, P. A., Boileau, N. R., Kratz, A. L., Hahn, E. A., \& Carlozzi, N. E. (2019). Sociocultural factors influencing caregiver appraisals following traumatic brain injury. Archives of Physical Medicine and Rehabilitation, 100(4), S58S64. https://doi.org/10.1016/j.apmr.2018.08.193

Sedgwick, T. W. (2012). Early hospital social work practice: The life and times of Janet Thornton. Affilia, 27(2), 212-221. https://doi.org/10.1177/0886109912444103

Taylor, C. A., Bell, J. M., Breiding, M. J., \& Xu, L. (2017). Traumatic brain injury-related emergency department visits, hospitalizations, and deaths - United States, 2007 and 2013. MMWR Surveillance Summaries, 66(9), 1-16. https://doi.org/10.15585/mmwr. $\underline{\text { ss6609a1 }}$

Thornton, M., \& Travis, S. S. (2003). Analysis of the reliability of the modified Caregiver Strain Index. Journals of Gerontology Series B: Psychological Sciences and Social Sciences, 58(2), S127-S132. https://doi.org/10.1093/geronb/58.2.s127

Turner, B., Fleming, J., Parry, J., Vromans, M., Cornwell, P., Gordon, C., \& Ownsworth, T. (2010). Caregivers of adults with traumatic brain injury: The emotional impact of transition from hospital to home. Brain Impairment, 11(3), 281-292. https://doi.org/10.1375/brim.11.3.281

Author note: Address correspondence to Kristen Linton, Health Science Program, California State University Channel Islands, One University Drive, Camarillo, CA 93012. Email: Kristen.linton@csuci.edu. 